\section{KOMPASS}

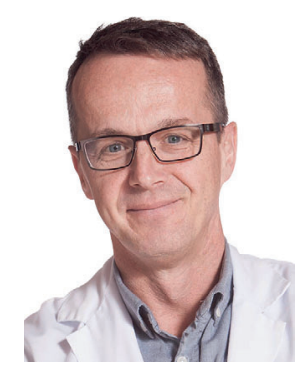

\section{Marc Spielmanns}

Zürcher RehaZentrum Wald, Schweiz

\title{
Lebensqualität erhalten und verbessern
}

Die Volkskrankheit COPD - primär eine Erkrankung der Bronchien und der Lungen zieht in der Regel eine Reihe von Begleitproblemen außerhalb der Lunge nach sich, welche nicht nur den Krankheitsverlauf, sondern auch die Lebensqualität der Betroffenen negativ beeinflussen. Sie ist damit eine Herausforderung für alle an der Therapie beteiligten Berufsgruppen. Gerade psychische Störungen beeinträchtigen die Lebensqualität von Erkrankten erheblich und stellen die Behandler oft vor große Probleme, weil jedwede therapeutische Intervention nicht zu helfen scheint. Gemäß einer aktuellen Studie weisen aber etwa 40\% der COPD-Patienten eine erhöhte Depressivität auf und etwa 36\% leiden unter einer erhöhten Ängstlichkeit. Außerdem konnte gezeigt werden, dass das Ausmaß von Angst und Depression nicht unbedingt mit der Schwere der COPD zusammenhängt. Sie zeigte sich im Stadium I und II mit etwa 40\% ebenso häufig wie im Stadium III (38\%) und im Stadium IV (40\%).

Viele Patienten leiden unter einzelnen Depressions- oder Angstsymptomen, weshalb die Diagnosestellung Schwierigkeiten bereiten kann. Vor dem Hintergrund der gesundheitlichen Bedrohung durch die Erkrankung treten häufig auch «krankheitsbezogene Ängste» auf. Damit sind die Ängste und Sorgen der Betroffenen vor der Symptomatik selbst oder auch vor deren
Folgen für die Zukunft gemeint. Sind die Sorgen und Ängste in Bezug auf die Erkrankung jedoch sehr stark ausgeprägt, können sie selbst zu einer Belastung werden und die Lebensqualität der Betroffenen zusätzlich beeinträchtigen. So kann Angst vor Atemnot dazu führen, dass die Betroffenen körperliche Aktivität vermeiden. Ein sich gegenseitiges Bedingen von körperlichem (Atemeinschränkung) und psychischem (Not beim Atmen) Befinden erscheint deshalb nicht überraschend. Atemnot, Angst und Depression können sich im Verlauf einer fortschreitenden COPD im Sinne eines sich selbst unterhaltenden Teufelskreises verselbstständigen. Die Betroffenen ziehen sich in der Folge immer mehr vom Alltagsund sozialen Leben zurück, was wiederum zu weiteren Beeinträchtigungen führen kann. Studien zeigen, dass dadurch nicht nur die Lebensqualität sinkt, sondern auch die Anzahl an Krankenhausbehandlungen und die Infektanfälligkeit steigen.

Trotz der Häufigkeit psychischer Probleme werden diese immer noch zu selten zwischen Ärzten und Patienten angesprochen. Es finden sich Studien, bei denen kein Patient jemals mit den behandelnden Ärzten über seine Ängste gesprochen hat.

Die eigenen Erfahrungen zeigen, dass auf psychische Probleme angesprochene Patienten die angebotene Hilfestellungen (z.B. eine Gesprächstherapie mit einem Psycho- logen im Rahmen einer pneumologischen Rehabilitation) zum Teil vehement ablehnen, da sie ja nicht als «verrückt» gelten wollen. Andererseits zeigt die klinische Erfahrung aber auch, dass bereits kleine Maßnahmen zu einer Abnahme von Angst und Depression führen können. Ein wichtiger Schritt in die richtige Richtung ist als Therapeut von Betroffenen erst einmal, daran zu denken und diese Themen anzusprechen. Über Zukunftsängste zu sprechen und Vorschläge z.B. in der Betreuung aufzuzeigen, entlastet die Patienten. Die multimodale pneumologische Rehabilitation bietet hierbei die ideale Plattform für die Patienten. Alle dafür erforderlichen Berufsgruppen können Hand in Hand an Lösungsansätzen arbeiten und damit ganz erheblich eine Steigerung der Lebensqualität erwirken. Im ersten Wissenstransfer zum Themenschwerpunkt dieser Ausgabe von KARGER Kompass PNEUMOLOGIE wird eindrucksvoll gezeigt, wie belastende Lebenssituationen auf den weiteren Verlauf der Symptomatik und der Lebensqualität von COPD-Patienten Einfluss nehmen. Im Wissenstransfer von Johannes Knoch wird die Nutzung der modernen Medien (Smartphone-App) auf das Selbstmanagement von COPD-Patienten beleuchtet. Axel Tobias Kempa kommentiert eine Studie, welche die Belastung von pleuralen Dauerkathetern für Krankenhäuser und Patienten untersuchte. Von gro-

\section{KARGER}

Fax +4976145207 14

information@karger.com

www.karger.com
๑๑ 2019 S. Karger GmbH, Freiburg

Accessible online at: www.karger.com/kkp
PD Dr. Marc Spielmanns

Zürcher RehaZentrum Wald

Faltigbergstrasse 7, 8636 Wald, Schweiz

marc.spielmanns@zhreha.ch 
ßem Interesse für Pneumologen dürften auch die Auswirkungen der zunehmenden Ausbreitung der Ambrosia als hochpotentes Allergen sein, die von Claudia TraidlHoffmann resümiert werden. Peter Haidl befasst sich in seinem Beitrag mit der Frage, ob die Bronchodilitator-Verneblung bei akuter Luftnot von COPD-Patienten mit Raumluft oder Sauerstoff erfolgen sollte. In der Übersichtsarbeit von Dan Adler und Jean-Paul Janssens werden die Wechselwirkungen zwischen der Atmungsregulation, der Atemlast und der Muskelfunktion dar- gestellt und in Bezug zur Ateminsuffizienz gestellt. Chieko Mitaka und Kollegen berichten in 2 interessanten Fällen über den erfolgreichen Einsatz der High-flow-Sauerstofftherapie im Rahmen des Weaningprozesses.

Und schließlich stellt Katrin Müller in der Rubrik Steckbrief Forschung ihre mit dem Doktorandenpreis 2018 der Deutschen Lungenstiftung ausgezeichneten Forschungen zu den physischen und psychischen Determinanten des Krankheitsmanagements von Patienten mit chronischen
Lungen- und Atemwegserkrankungen vor. Wir hoffen, Ihnen in diesem Heft aus der Fülle an aktuellen Veröffentlichungen eine interessante Zusammenstellung zum Themenschwerpunkt - aber auch abseits davon in den übrigen Beiträgen - zu präsentieren. Viel Freude beim Lesen wünscht Ihnen

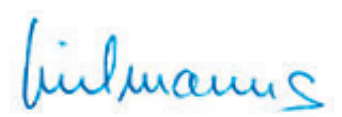

Ihr PD Dr. Marc Spielmanns 